\title{
Bridging the Adoption Gap for Smart City Technologies: An Interview with Rob Kitchin
}

\author{
Katja Schechtner, MIT Media Lab
}

T he concept of smart cities has become increasingly popular, but it seems more difficult than ever to bridge the gap between what city administrations want and what developers of smart city technologies offer. At the same time, citizens are trying to understand how these technologies will make their lives better.

Rob Kitchin, a professor and European Research Council Advanced Investigator at the National University of Ireland Maynooth, has more than 15 years of experience in addressing various aspects of smart city concepts to create solutions that work for cities and their citizens alike. He is (co)principal investigator of the Programmable City project, the Building City Dashboards project, the All-Island Research Observatory, and the Digital Repository of Ireland. Much of Kitchin's hands-on work concerns the development of urban dashboards-such as the Dublin Dashboard (see Figure 1) — that seek to collate all of the urban big data produced by city systems, along with traditional statistical and public administration data, and to visualize that data to make it actionable. Building on this knowledge, Kitchin's team is currently looking at the social, political, and economic implications of creating smart cities as part of a recent European Research Council grant, and how to build more effective city dashboards through a Science Foundation Ireland grant.

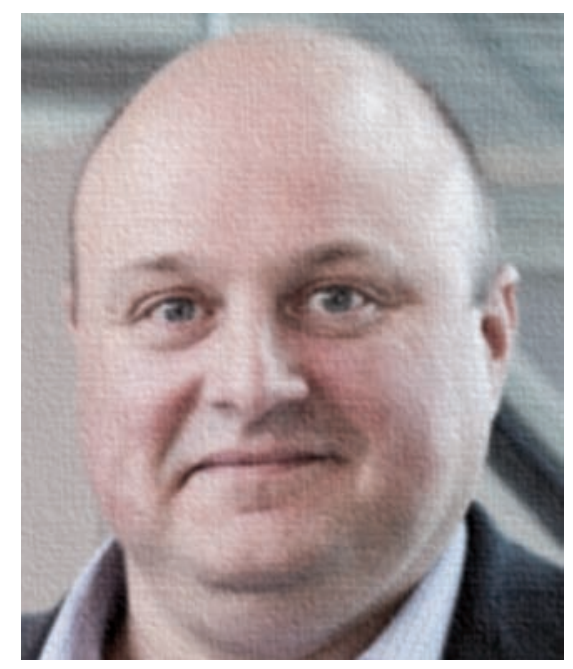

Rob Kitchin, 2013 recipient of the Royal Irish Academy's Gold Medal for the Social Sciences.

It's with this background in mind that I discussed with him whether architects and planners and electronic engineers and computer scientists have a different understanding of cities and, if so, how we might bridge the gap.

You have argued that dashboardsand smart city initiatives in generalare often underpinned by a naive instrumental rationality. What do you mean by this, and why is it an issue?

Smart city technologies mostly seem founded on a rationality that supposes that cities, and their various services and functions, can be steered and managed through a set of technical solutions. In other words, the various complex issues facing citizens and city managers can be disassembled into neatly defined technical problems that can be adequately solved through technology. All that is required to understand, manage, and fix urban issues in a rational, logical, and impartial way is a suitable technical kit, sufficient data, and clever algorithms. In this view, urban dashboards provide a set of data levers for steering the management of the city. The problem with this perspective is fourfold.

First, a technical approach reduces city systems and people to relatively simple components and agents. This mostly ignores the metaphysical aspects of human life; subjectivity; and the role of politics, ideology, soft values, social and institutional structures, capital, and culture in shaping everyday living and urban development and governance. As such, it's overly reductive and anemic in nature. This is exacerbated by a positioning of technology as neutral, objective, pragmatic, and commonsensical, rather than full of choices, values, and politics.

Second, the technical approach frames urban issues in instrumental and practical ways, rather than within a wider normative framework. So smart city technologies aim to solve questions such as how can we optimize traffic? How can we reduce energy usage? How can we more 
effectively police an area? How can we increase the efficiency of service delivery? The issues might be framed with respect to notions of sustainability, safety, security, economic competitiveness, consumer choice, and so on, but often in a shallow, limited sense. For example, developers might state that a technology can make a system more sustainable, without saying what "being sustainable" means beyond instrumental targets. There are many conceptions of sustainability, and adopting the principles of different positions might lead to the development of alternative solutions. Smart city initiatives then rarely start with deeper normative concerns with respect to fairness, equity, justice, citizenship, democracy, governance, political economy, and questions such as, "What kind of cities do we want to create and live in beyond a limited instrumental framing?"

Third, the technical approach assumes that technology can fix all of a city's issues, rather than acknowledging that some issues might be best solved through political or social interventions, collective action, public policy, investment in infrastructure, or citizen-centered deliberative democracy. There is often a "hammer and nail" mentality in the approach adopted-that is, "if one makes hammers, then all problems look like nails.” In turn, technological solutionism promotes technocratic governance that is narrowly and instrumentally focused and works in constrained and constraining ways.

Fourth, a technical approach can often produce what might be termed "sticking plaster solutions." For example, technical solutions to traffic congestion are often about trying to optimize flow or re-route vehicles. They don't address the deep-rooted problem that there are too many vehicles using the road system or provide a solution that shifts people onto public transport or encourages more cycling and walking. Similarly, we're not going to solve homelessness with an app. It's an issue of social inequalities and often mental

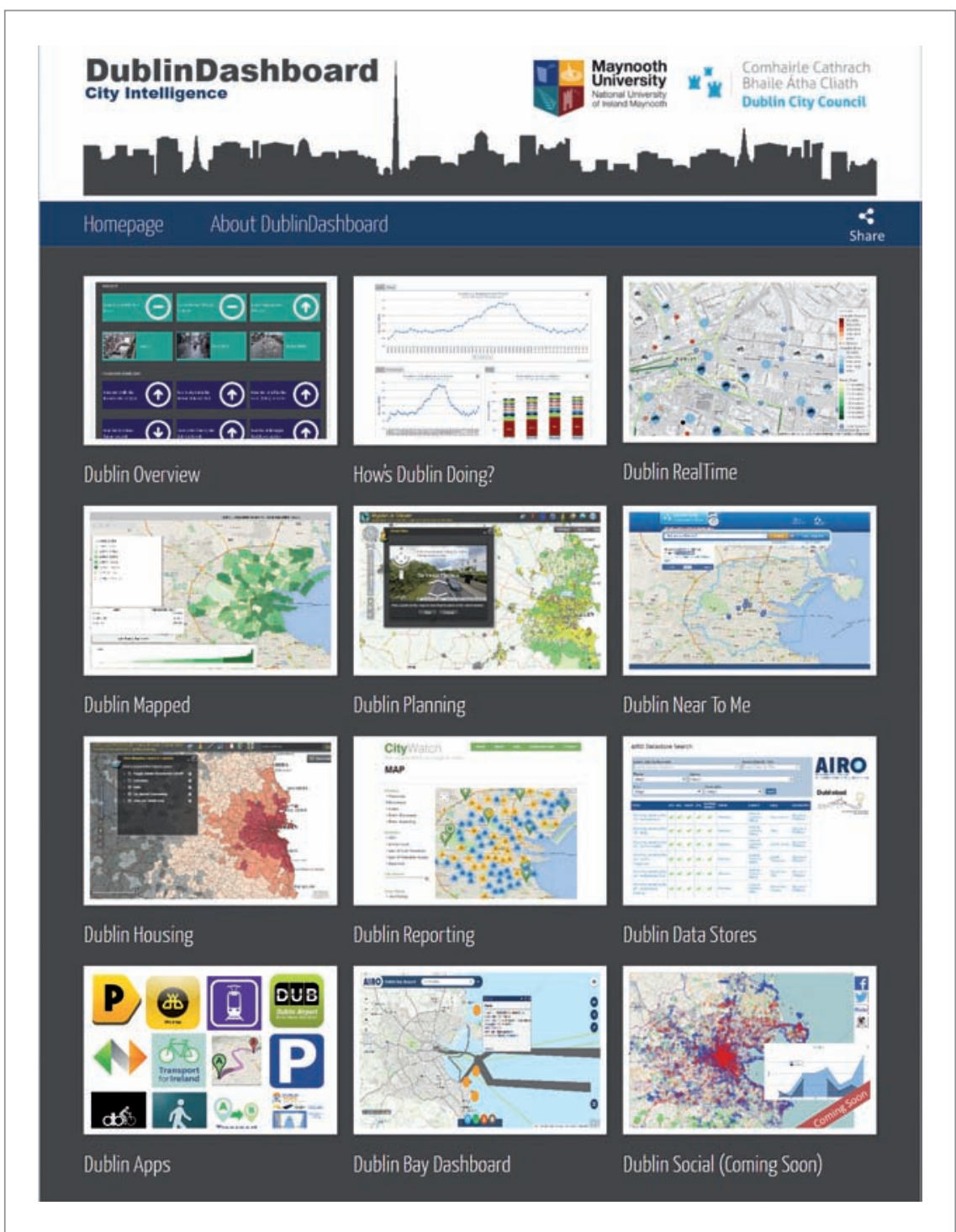

Figure 1. The Dublin Dashboard, which "provides citizens, public sector workers, and companies with real-time information, time-series indicator data, and interactive maps about all aspects of the city" (www.dublindashboard.ie/pages/ContactUs). Rob Kitchin is the principal investigator.

health, drug dependency, and social violence. An app might help manage homeless services more effectively, but it's not going to address the underlying structural causes.

What the instrumental rationality and associated criticisms of technology-led solutions to city issues mean is that urban planners and city managers are sometimes cautious about adopting them. This doesn't mean that such planners and managers are anti-technology; rather, they want the optimal solution to an issue, which may or may not involve technology or technology working in concert with other solutions. And they want the technology to be open about its underlying ideas, rationalities, logics, and limitations. For example, with the Dublin Dashboard, we have sought to be open about the aims, principles, praxes, and politics of the initiative and to think critically about how the dashboard influences urban governance.

So beyond an instrumental rationality, do architects and planners and 
electronic engineers and computer scientists have a different understanding of cities?

My impression is that electronic engineers and computer scientists tend to see the city as a set of knowable and manageable systems-or system of systems-that act in largely rational, mechanical, linear, and hierarchical ways. In addition, city systems are largely treated as generic analytical categories with some typical variances, meaning a solution developed for one city can be transferred and replicated elsewhere. And while cybernetic approaches recognize the complexity and emergent qualities of city systems, they're still understood as being machinic and largely closed and bounded in nature. This system view of cities is a narrow conception of what a city is and how it works.

In contrast, planners and city administrators understand the city as being complex, multifaceted, contingent, open and relational, and full of contestation and wicked problems. They typically see cities as places, not systems. From this position, cities have different histories, cultures, social and community relations, economies, governance structures, institutional structures, politics, legacy infrastructures, political and administrative geographies, and interconnections and interdependencies with other places. Cities have a range of different, often competing, actors and stakeholders-government bodies, public sector agencies, companies, nongovernmental bodies, community organizations, and so on-that have different goals, resources, practices, and structures and that are trying to address and manage various issues. This messiness isn't well captured in computational logic and is difficult to model, predict, and manage through technocratic governance. Understanding cities from this perspective, it seems clear that smart city technology won't be a silver bullet to urban issues.

Unlike scientists and engineers, who are usually excited about new or improved methods and tools for planning, monitoring, and managing cities, there seems to be a lack of interest and excitement on the part of urban planners and architects. Why is this?

Beyond concerns related to technological solutionism and instrumental rationality, there are a number of reasons that cities are cautious with respect to smart city technologies.

The first reason is risk. A city manager will tell you that his or her job is to provide stability, certainty, and reliability in the delivery of city services. A lot of smart city technology is not mature. That is why there is a boom in what has been called "experimental" or "testbed" urbanism or "living labs." Technologies are still being developed and tested. They are like drugs in the clinical trial phase. Unless there is a compelling reason to be a first mover, perhaps because a problem is so acute that it's worth taking a risk, or a city is trying to gain a competitive advantage related to economic development, then the city manager would prefer to exploit the second-mover advantage - that is, the advantage of knowing the system will work in solving a particular problem and improve city services. For example, if city management is going to upgrade 50,000 lampposts to smart lighting, they want to know the system is going to work well and do what was promised. They don't want a newspaper headline that states, "\$15 Million of Taxpayers" Money Wasted."

Another reason, which is also related to risk, is trust. Planners and city administrators need to trust that new initiatives will work. They have a long history of purchasing technologies that are costly and don't always deliver on their promises. This includes the wave of first-order urban cybernetic systems in the 1970s that failed horribly and were widely critiqued and abandoned. In fact, the move toward a technocratic approach at that time created a strong backlash in the planning profession, moving it to a much more collaborative, participatory approach. Some smart city technology aims to foster such a citizen-led approach by crowdsourcing data and opinions and fostering debate, but much of the technology is rooted in second-order cybernetics and other technocratic governance approaches.

A third concern relates to the amount of perceived value for money spent and the return on investment. Many technological solutions are not cheap, and it isn't always clear what the return on investment will be beyond promises that an issue will be ameliorated in some way. Moreover, it's clear that the same technology will be cheaper and better-in terms of spec, functionality, performance-in a few years, so it's difficult to know when to make the initial investment. Many cities are currently operating in a condition of austerity, so finances for new investments are constrained. As such, although some technologies could save the city money over the long term, the city still must find the initial investment capital. This is why so much effort is now being expended on new business models for smart city investments.

Another issue is competing demands. City administrations are responsible for managing a range of infrastructures and services. There are many competing demands for a limited budget, and many of these are statutory obligations. Unless a proposed solution will solve a critical problem, rather than merely offer a nice enhancement, it will have trouble competing for attention and resources. What smart city technology developers versus city administrations view as critical issues can be quite divergent.

In addition, city administrations are overloaded. Many stakeholders underappreciate the extent to which they are being bombarded by companies, consultants, lobbyists, academics, and so on, all seeking attention for their smart city technology. It can be quite difficult to work out from the noise which technology is worth pursuing. City administrations must be selective; they can't pursue all possible initiatives. 
The final issue is inertia and resistance. Like all big organizational entities, city administrations have existing practices and legacy systems and are full of internal politics, fiefdoms, and competing interests. Workers can be reluctant to upset the status quo unless what is being proposed is going to substantially improve existing workflows or provide a better solution. In some cases, these "better solutions" will be resisted, especially if they will lead to substantial job cuts.

To promote an understanding between the two groups, architects and urban planners are usually advised to learn a programming language to understand the thinking of "the other side." What would your advice be for electrical engineers and computer scientists who would like to work with cities?

Rather than start with your question, let's start with your initial statement. Why should architects and planners learn how to code? Let's answer that with two questions: Should patients train as medics to understand doctors? Should the users of smartphone apps learn to code to use those apps? Or should the doctors be able to explain their diagnosis in a way that patients can understand and trust, and should the app be intuitive to use and have suitable help support? There is almost a "blame the victim" mentality in the argument that architects and planners should learn to code, because electronic engineers and computer scientists can't make their rationalities, imaginaries, logics, and systems intelligible, or convince people that their solutions are better than others.

Architects' and planners' work is to create, build, and plan cities. That involves certain kinds of specialist knowledges that take years of training and experience to develop. Similarly, electronic engineers and computer scientists learn specialist knowledge to produce infrastructure and computational systems. Yes, they have pragmatic knowledge of cities based on living in them, but that doesn't make them experts with respect to architecture, urban design, planning principles, transport systems, social issues, legal and regulatory conditions, and the long history of various kinds of interventions-both policy and practical-previously used to try and solve long-standing issues. So, should electronic engineers and computer scientists train as architects, planners, and other domain specialisms before they start to create technical solutions for city problems? Should they understand in depth the long history of previously attempted solutions and why they are suboptimal? Or should they just work with people who already have this knowledge?

The solution to the gap in knowledge about how particular specialists approach urban issues is communication and mutual learning, not training to gain the core competencies of the other. It's about working together in teams. It's about doing full requirements analysis informed from all sides. It's about respecting each other's perspectives and approaches-and there are also significant differences in epistemology, ontology, methodology, and ethos across disciplines-and accommodating different viewpoints and knowledge. In our work on urban dashboards, this is how we work; we have a mixed team of social scientists and researchers who are from computer science, data science, and geoscience, as well as having a partnership with six local governments and their domain practitioners and Ordnance Survey Ireland [the national mapping agency] and the Central Statistics Office.

As such, there is a mix of academic and technical expertise and many years of practical experience of trying to address issues on the ground. In other words, we are trying to blend episteme (scientific knowledge), teche (practical instrumental knowledge), phronesis (knowledge derived from practice and deliberation), and metis (knowledge based on experience). We're also not just interested in building dashboards but also in asking technical/practical questions related to data quality and data access. How will the dashboards create particular views of the world, how will they be used in practice to make decisions and to do political work, and what will be the consequences and ethics of that work? We use a very plural approach that draws on a range of philosophical positions, which we try to frame more normatively.

So, my advice to electrical engineers and computer scientists is to do two things. First, build interdisciplinary/ domain practitioner teams that are genuinely interdisciplinary; not teams that are heavily science/engineering dominated with token social scientists that have very limited roles and responsibilities.

Second, understand the critiques levelled at technical approaches to solving urban issues and constraints that are faced by city managers and try to find ways to accommodate and work around them. My experience is that people working for city governments genuinely want to improve the quality of life of their citizens, institutions, and companies located there. If a smart city solution will help them do that, while also mitigating against constraints, then they are interested. But they have to be convinced that the final solution will work with few unanticipated negative consequences, and the value must be worth the investment. $\mathbf{E}$

Katja Schechtner is a visiting scholar at the MIT Media Lab and a visiting professor at the Vienna University of Technology. Contact her at katjas@mit. edu.

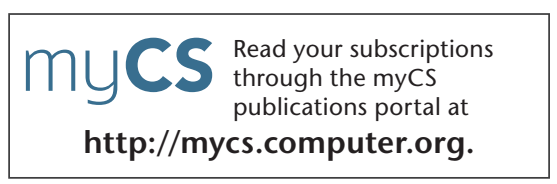

